\title{
What Influences Public History the Most
}

public-history-weekly.degruyter.com/7-2019-19/historical-beliefs/

Gautschi, Peter

May 23, 2019

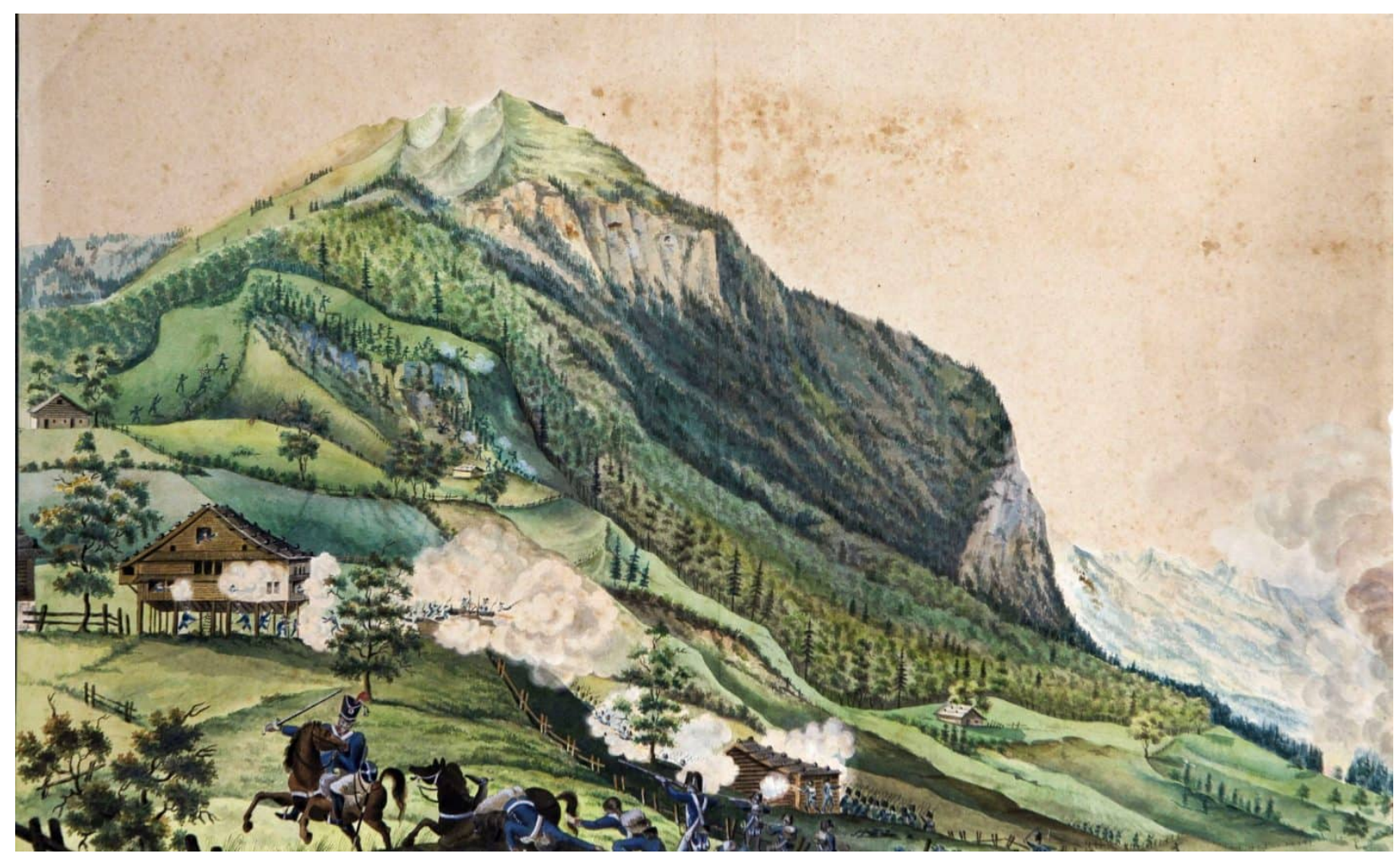

- English

- Deutsch

As regards the communication of history in schools we have suspected it for a long time: It is the history-related beliefs of teachers that make the difference. The communication of history in public as well is less influenced by scientific findings than by the historical beliefs of the people involved. In contrast to schools where teachers often alone decide on how to convey history and memory, this is, however, mostly negotiated when it comes to the communication of history in public - as becomes clear from the example of the history trail "French Invasion".

\section{The "French Invasion" in Nidwalden of 1798}

In Switzerland as well the French Revolution had a significant impact. The ideas of the Enlightenment were enthusiastically received in many places. Revolutionary uprisings in different places against the old order of the Swiss Confederation gave the French troops in 1798 a reason to occupy the territory and to proclaim the "Helvetic Republic". But the new order was not accepted everywhere at all. When the men who had the voting rights in Nidwalden were supposed to swear the oath of allegiance to the new Helvetic Constitution, a popular uprising broke out, which led to military clashes. On 9 September 1798 just under 1,600 natives of 
Nidwalden threw themselves into a hopeless battle against a 10,000 men strong French army under General von Schauenburg.[1] When the French managed to break through at the Allweg, the fight was decided.[2]

The great resistance of the inhabitants of Nidwalden now, amongst other things, caused the victorious French to rage in the region, to pillage it, to violate women and to massacre people after the fight. The priests who had organized the resistance were murdered along with many civilians, all in all roughly 5 percent of the population of Nidwalden.[3] The great hardship led to the fact that the Enlightenment pedagogue Johann Heinrich Pestalozzi received the order to set up an orphanage and poorhouse in Stans.[4] Here he made fundamental pedagogical experiences which he then used to found his famous education institute the following year at Burgdorf Castle where he developed and theoretically substantiated a teaching and education method of his own.[5]

\section{Three Ways of Teaching History in Schools}

All these events surrounding the "French invasion" of 1798 can be imparted completely differently in history lessons. Some of the teachers intend to impart knowledge: They narrate and explain how things were in Nidwalden at the time, what happened and in what order, what had been the causes and consequences. Other teachers want to encourage students to think in historical-scientific terms and make them compare multi-perspective sources and controversial representations as well as to critically discuss questions: Why did the people of Nidwalden not restrain from becoming involved in such hopeless fighting? Were they misled and manipulated? In such a teaching the focus lies on the competent dealing with materials from and about the past. It is all about the students' ability and critical thinking. And still other teachers wish to build up attitudes: They stage dilemma situations, provide case studies, make comparison with today and accompany students on their way to the insight that also these days it is important to stand up for the victims of war.

\section{History-related Beliefs}

What teachers wish to achieve in their history lessons and how they stage their teaching is marked by their history-related beliefs. [6] More recent research on teachers' subject-specific beliefs in German-speaking Europa identify three manifestations:[7] Martin Nitsche distinguishes positivism, skepticism and narrative constructivism;[8] Nicole Riedweg, Peter Gautschi and Loredana Torchetti differentiate between "history as a master teacher for the present", "history as a science" and "history as a narrative about the past".[9]

In the communication of history in public other beliefs can be recognized. This is because the communication of history in public is more strongly driven by trends than the communication of history in schools. Thus, "identity" plays a major role in particular. Whoever wants to convey the message "Be like us!" emphasizes either the heroic defensive battle of the people of Nidwalden or the standing up for Enlightenment ideas.[10] Whoever wishes to entertain through history picks out emotionally touching episodes from these events, and stages them, for example, in a radio play or with augmented reality. History can also help to keep alive the memory of relatives or compatriots killed in action and to grieve for them. And, of course, history, every now and 
then, serves as a place of longing, as a possibility to get out of the, sometimes boring, everyday life and to escape into an era in which exciting radical changes and interesting upheavals happened.

\section{Individual and Common Beliefs}

If now a history trail shall be set up at the site of where the armed clashes took place during the "French invasion" - in Stansstad in the Swiss canton of Nidwalden - the people involved have to discuss their perceptions and history-related beliefs with each other, to negotiate and align them. The contracting authorities have, of course, a great influence on all the communication of history in public. When it comes to history trails this is mostly the executive authority of the municipality on whose territory such a history trail is established. If the arising expenses exceed the amount which the executive authority is entitled to allow, the legislative authority must decide. In Switzerland at municipal level these are oftentimes all the voters either at the ballot box or otherwise at the municipal assembly at a designated place of assembly. This, again and again, triggers exciting discussions, first of all, about the motives why such a history trail should be made, secondly, about the target groups and, thirdly, about the goals which should be accomplished with the history trail. And, at this point, beliefs as to what the purpose of history is and what it can achieve play the greatest role. Ideally, a commonly shared belief about the goals and the staging is built by this exchange in the community. If a consensus can be found, the concrete realization at the very site mostly causes no problem, and the history trail is accepted by the local population and also used by visitors - as an experience and for history education. [11]

\section{Further Reading}

- Fives, Helenrose, and Michele Gregoire Gill, eds. International Handbook of Research on Teachers' Beliefs. New York: Routledge, 2014.

- Gautschi, Peter, Armin Rempfler, Barbara Sommer Häller and Markus Wilhelm, eds. Aneignungspraktiken an außerschulischen Lernorten. Münster et al.: LIT Verlag, 2018.

- Odermatt, Katharina. "Der lange Weg zum modernen Staatswesen." In Geschichte des Kantons Nidwalden: Band 1, edited by Peter Steiner, Hansjakob Achermann, Karin Schleifer-Stöckli and Emil Weber, 127-140. Stans: Verlag Historischer Verein Nidwalden, 2014.

\section{Web Resources}

- Website of the legionnaire path of Vindonissa (Museum of Aargau). https://www.ag.ch/legionaerspfad.jsp (last accessed 17 April 2019).

- Path of Morgarten. http://www.morgarten.ch/erleben/ (last accessed 17 April 2019).

- Erster Wiener Protestwanderweg: https://www.protestwanderweg.at/ (last accessed 17 April 2019) 
[1] For a history of events, see Martin Illi, "Franzoseneinfall," Historisches Lexikon der Schweiz (HLS), December 17, 2009, http://www.hls-dhs-dss.ch/textes/d/D8915.php (last accessed 17 April 2019).

[2] Katharina Odermatt, "Der lange Weg zum modernen Staatswesen," Geschichte des Kantons Nidwalden: Band 1, ed. Peter Steiner, Hansjakob Achermann, Karin Schleifer-Stöckli and Emil Weber (Stans: Verlag Historischer Verein Nidwalden, 2014), 127-140.

[3] Christoph Baumgartner, "Der 'Franzosenüberfall' von 1798," State Chancellery of the Canton of Nidwalden, October 2016, https://www.nw.ch/ docn/86826/Geschichte_Franzosenueberfall_2016 10.pdf (last accessed 17 April 2019).

[4] See e.g. Wolfgang Klafki, "Pestalozzis 'Stanser Brief'. Eine Interpretation," in Pestalozzi über seine Anstalt in Stans. Mit einer Interpretation und neuer Einleitung von Wolfgang Klafki, ed. Wolfgang Klafki (Weinheim: Beltz, 1997), 39-71. [5] See also Peter Stadler, Pestalozzi: Geschichtliche Biographie (Two volumes; Zurich: Verlag NZZ, 1988 and 1993) or Bijan Amini, Johann Heinrich Pestalozzi: Einführung in Leben und Werk (Pinneberg: Heseberg Verlag, 2018).

[6] Carla L. Peck and Lindsay Herriot, "Teachers' beliefs about social studies," in International Handbook of Research on Teachers' Beliefs, eds. Helenrose Fives and Michele Gregoire Gill (New York: Routledge, 2014), 387-402. It becomes apparent in this handbook also that it is no simple undertaking to define what "history-related beliefs" exactly are. It seems solely clear that they are relatively stable, judgmental and powerful opinions as to what history is and what purpose it serves.

[7] The studies with their three manifestations each remind of the differentiation made by Bernd Schönemann: "History as a benefit", "history as education" and "history as an experience." For further information, see Bernd Schönemann, "Geschichtsdidaktik und Geschichtskultur," in Geschichtskultur: Theorie - Empirie - Pragmatik, eds. Bernd Mütter, Bernd Schönemann and Uwe Uffelmann (Weinheim: Beltz, 2000), 26-58, here 47-55.

[8] Martin Nitsche, Beliefs von Geschichtslehrpersonen: Eine Triangulationsstudie (Bern: Hep Verlag, to be published in 2019). Nitsche refers to positivism, skepticism and narrative constructivism as "historical-theoretical beliefs". But other than that, he from the former distinguishes three "historical-didactic beliefs": Transmission, individual constructivism, social constructivism. In Nitsche's study it seems particularly interesting to me that no systematic cross-cutting constructional connections between the historical-theoretical and historical-didactic beliefs could be found.

[9] Nicole Riedweg, Peter Gautschi and Loredana Torchetti, "Fachspezifische Überzeugungen von Geschichtslehrpersonen," in The Teaching of the History of One's Own Country, eds. Nadine Fink, Markus Furrer and Peter Gautschi (Frankfurt: Wochenschau Verlag, to be published in 2020). With the questionnaire data collected in this study a model with two manifestations could also be formed: History as an orientation for the present and the future / history as a construct of the past.

[10] Identity building also exists in a less pronounced way, rather in the sense of tradition building. See also Astrid Schwabe and Martin Fröhlich, "Erinnerungen an 1918 in Kiel: Schlaglichter auf die regionale Geschichtskultur im Gedenkjahr 2018," Demokratische Geschichte: Jahrbuch für Schleswig-Holstein 29 (2018): 171-197.

[11] In contrast to the communication of history in schools where the concept of "education" has over the last few years been replaced by the concept of "competences", "history education" ("Historische Bildung") has remained the determining orientation in the German-speaking public history. People are thus historically educated if they have differentiated personal and social identities, who openly and curiously encounter the vast field of history, who have welldeveloped competences when dealing with the past, history and memory and who, based on that, recognize and use their own scopes for action in the present and future as well as they are aware of the opportunities of history education and want to continue to educate themselves.

\section{Image Credits}

Louis Victor von Deschwanden: Kampf am Allweg (probably between 1800 and 1820) @ Courtesy of the Museum of Nidwalden (NM 514). 
Recommended Citation

Gautschi, Peter: What Influences Public History the Most. In: Public History Weekly 7 (2019) 19, DOI: dx.doi.org/10.1515/phw-2019-13911(P).

Translated by Kurt Brügger swissamericanlanguageexpert https://www.swissamericanlanguageexpert.ch/

Editorial Responsibility

Judith Breitfuß / Thomas Hellmuth (Team Vienna)

In der schulischen Geschichtsvermittlung vermuten wir es schon lange: Es sind die geschichtsspezifischen Überzeugungen der Lehrer*innen, die den Unterschied machen. Auch die öffentliche Geschichtsvermittlung wird weniger durch wissenschaftliche Erkenntnisse, sondern durch Überzeugungen der Beteiligten beeinflusst. Im Unterschied zur Schule, wo die Lehrer*innen oft allein bestimmen, wie sie Geschichte und Erinnerung vermitteln, wird dies in der öffentlichen Geschichtsvermittlung meist ausgehandelt, wie am Beispiel des Geschichtswegs "Franzosenüberfall" deutlich wird.

\section{Der “Franzosenüberfall” in Nidwalden von 1798}

Auch in der Schweiz hatte die Französische Revolution erhebliche Auswirkungen. Die Ideen der Aufklärung wurden mancherorts begeistert aufgenommen. Revolutionäre Aufstände an verschiedenen Orten gegen die alte Ordnung der Eidgenossenschaften lieferten den französischen Truppen 1798 einen Grund, das Gebiet zu besetzen und die "Helvetische Republik" auszurufen. Die neue Ordnung wurde aber keineswegs überall akzeptiert. Als die stimmfähigen Männer in Nidwalden den Treueeid auf die neue helvetische Verfassung schwören sollten, brach ein Volksaufstand aus, was zu kriegerischen Auseinandersetzungen führte. Am 9. September 1798 stürzten sich knapp 1600 Nidwaldner in eine aussichtslose Schlacht gegen die rund 10.000 Mann starke französische Armee unter General von Schauenburg.[1] Als den Franzosen der Durchbruch am Allweg gelang (siehe Bild), war der Kampf entschieden.[2]

Nun führte aber der große Widerstand der Nidwaldner unter anderem dazu, dass die siegreichen Franzosen nach dem Kampf in der Gegend wüteten, brandschatzten, vergewaltigten, massakrierten. Ermordet wurden Priester, die den Widerstand organisiert, und auch viele Zivilist*innen, insgesamt rund fünf Prozent der Nidwaldner Bevölkerung.[3] Die große Not bewirkte, dass der aufklärerische Pädagoge Johann Heinrich Pestalozzi den Auftrag bekam, in Stans ein Waisen- und Armenhaus einzurichten.[4] Hier machte er grundlegende pädagogische Erfahrungen, die er nutzte, um im darauf folgenden Jahr im Schloss Burgdorf sein berühmtes Erziehungsinstitut zu gründen, wo er eine eigene Unterrichts- und Erziehungsmethode entwickelte und theoretisch begründete.[5]

\section{Drei Vermittlungsrichtungen in der Schule}

Diese Geschehnisse rund um den "Franzosenüberfall von 1798" können im Geschichtsunterricht ganz unterschiedlich vermittelt werden. Die einen Lehrer*innen wollen Wissen vermitteln: Sie erzählen und erklären, wie es damals in Nidwalden war, was in welcher Reihenfolge geschah, was die Ursachen und die Folgen gewesen sind. Andere Lehrer*innen 
wollen die Schüler*innen zum geschichtswissenschaftlichen Denken hinführen und lassen sie multiperspektivische Quellen und kontroverse Darstellungen vergleichen sowie Fragen kritisch diskutieren: Wieso haben sich die Nidwaldner*innen in solch aussichtslose Kampfhandlungen verwickeln lassen? Wurden sie verführt und manipuliert? In solch einem Unterricht steht der kompetente Umgang mit Materialien aus der und über die Vergangenheit im Zentrum. Es geht um das Können und um das kritische Denken der Schüler*innen. Und wieder andere Lehrer*innen wollen Einstellungen aufbauen: Sie inszenieren Dilemmasituationen, bieten Fallstudien an, machen Vergleiche zu heute und begleiten Schüler*innen auf ihrem Weg zur Einsicht, dass es auch heute wichtig ist, sich für Kriegsopfer einzusetzen.

\section{Geschichtsspezifische Überzeugungen}

Was die Lehrer*innen mit ihrem Geschichtsunterricht erreichen wollen und wie sie inn inszenieren, wird durch ihre geschichtsspezifischen Überzeugungen geprägt.[6] Neuere Forschungen im deutschsprachigen Europa zu fachspezifischen Überzeugungen von Geschichtslehrerinnen und -lehrern gehen von drei Ausprägungen aus:[7] Martin Nitsche unterscheidet Positivismus, Skeptizismus und Narrativen Konstruktivismus;[8] Nicole Riedweg, Peter Gautschi und Loredana Torchetti unterscheiden "Geschichte als Lehrmeisterin für die Gegenwart", "Geschichte als Wissenschaft" und "Geschichte als Erzählung über die Vergangenheit".[9]

In der öffentlichen Geschichtsvermittlung sind weitere Überzeugungen zu erkennen. Das hängt damit zusammen, dass öffentliche Geschichtsvermittlung stärker trendgetrieben ist als schulische Geschichtsvermittlung. So spielt insbesondere "Identität" eine große Rolle. Wer die Botschaft "Sei wie wir!" vermitteln will, hebt entweder den heldenhaften Verteidigungskampf der Nidwaldner oder das Einstehen für die aufklärerischen Ideen hervor.[10] Wer mit Geschichte unterhalten will, sucht emotional berührende Episoden aus diesen Geschehnissen heraus, und inszeniert sie beispielsweise in einem Hörspiel oder mit Augmented Reality. Geschichte kann auch mithelfen, das Gedenken an die gefallenen Verwandten und Landsleute wachzuhalten und um sie zu trauern. Und natürlich dient Geschichte ab und zu als Sehnsuchtsort, als Möglichkeit, um aus der altäglichen, vielleicht langweiligen Lebenswelt auszubrechen und in eine Zeit zu fliehen, in der es spannende Umbrüche und interessante Umwälzungen gab.

\section{Individuelle und gemeinschaftliche Überzeugungen}

Wenn jetzt am Ort der Auseinandersetzungen während des "Franzosenüberfalls" - in Stansstad im Schweizer Kanton Nidwalden - ein Geschichtsweg entstehen soll, müssen die Beteiligten ihre Vorstellungen und geschichtsspezifischen Überzeugungen miteinander diskutieren, verhandeln und abgleichen. Einen großen Einfluss hat natürlich bei allen Geschichtsvermittlungen in der Öffentlichkeit der Auftraggeber bzw. die Auftraggeberin. Bei Geschichtswegen ist dies meist die Exekutive derjenigen Gemeinde, auf deren Gebiet ein solcher Geschichtsweg entsteht. Übersteigen die anfallenden Kosten den Betrag, den die Exekutive selber tragen kann, muss die Legislative entscheiden. Dies sind in der Schweiz auf Ebene der Gemeinde oftmals alle Stimmbürger*innen an der Urne oder aber in der Gemeindeversammlung an einem ausgewählten Versammlungsort. Das führt immer wieder zu spannenden Diskussionen, erstens über die Motive, wieso ein solcher Geschichtsweg entstehen soll, zweitens über die Zielgruppen und drittens über die Ziele, die mit dem Geschichtsweg erreicht werden sollen. Und hier spielen die Überzeugungen, wozu Geschichte 
dient und was sie leisten kann, die größte Rolle. Im Idealfall baut sich durch den Austausch in der Gemeinschaft eine gemeinsam geteilte Überzeugung zu den Zielen und zur Inszenierung auf. Wenn ein Konsens gefunden wird, ist meist die konkrete Umsetzung vor Ort problemlos, und der Geschichtsweg wird von der Bevölkerung akzeptiert und auch von Besucher*innen genutzt - als Erlebnis und zur historischen Bildung.[11]

\section{Literaturhinweise}

- Fives, Helenrose, and Michele Gregoire Gill, eds. International Handbook of Research on Teachers' Beliefs. New York: Routledge, 2014.

- Gautschi, Peter, Armin Rempfler, Barbara Sommer Häller and Markus Wilhelm, eds. Aneignungspraktiken an außerschulischen Lernorten. Münster et al.: LIT Verlag, 2018.

- Odermatt, Katharina. "Der lange Weg zum modernen Staatswesen." In Geschichte des Kantons Nidwalden: Band 1, edited by Peter Steiner, Hansjakob Achermann, Karin Schleifer-Stöckli and Emil Weber, 127-140. Stans: Verlag Historischer Verein Nidwalden, 2014

\section{Webressourcen}

- Website des Legionärspfads Vindonissa (Museum Aargau): https://www.ag.ch/legionaerspfad.js $\underline{\text { p }}$ (letzter Zugriff 17. April 2019).

- Morgartenpfad: http://www.morgarten.ch/erleben/ (letzter Zugriff 17. April 2019).

- Erster Wiener Protestwanderweg: https://www.protestwanderweg.at/ (letzter Zugriff 17. April 2019)

[1] Zur Ereignisgeschichte, vgl. Martin Illi, "Franzoseneinfall," Historisches Lexikon der Schweiz (HLS), December 17, 2009, http://www.hls-dhs-dss.ch/textes/d/D8915.php (letzter Zugriff 17. April 2019).

[2] Katharina Odermatt, "Der lange Weg zum modernen Staatswesen," Geschichte des Kantons Nidwalden: Band 1, ed. Peter Steiner, Hansjakob Achermann, Karin Schleifer-Stöckli and Emil Weber (Stans: Verlag Historischer Verein Nidwalden, 2014), 127-140.

[3] Christoph Baumgartner, "Der ‘Franzosenüberfall' von 1798," State Chancellery of the Canton of Nidwalden, October 2016, https://www.nw.ch/ docn/86826/Geschichte Franzosenueberfall 2016 10.pdf (letzter Zugriff 17. April 2019).

[4] Vgl. dazu z.B. Wolfgang Klafki, "Pestalozzis 'Stanser Brief'. Eine Interpretation,” in Pestalozzi über seine Anstalt in Stans. Mit einer Interpretation und neuer Einleitung von Wolfgang Klafki, ed. Wolfgang Klafki (Weinheim: Beltz, 1997), 39-71.

[5] Siehe auch Peter Stadler, Pestalozzi: Geschichtliche Biographie (Two volumes; Zurich: Verlag NZZ, 1988 and 1993) oder Bijan Amini, Johann Heinrich Pestalozzi: Einführung in Leben und Werk (Pinneberg: Heseberg Verlag, 2018).

[6] Carla L. Peck and Lindsay Herriot, "Teachers' beliefs about social studies," in International Handbook of Research on Teachers' Beliefs, eds. Helenrose Fives and Michele Gregoire Gill (New York: Routledge, 2014), 387-402. Auch in diesem Handbuch wird deutlich, dass es kein leichtes Unterfangen ist zu definieren, was genau "geschichtsspezifische Überzeugungen" sind. Klar scheint immerhin, dass es relativ stabile, urteilsgeladene und wirkmächtige Meinungen sind, was Geschichte ist und wozu sie dient.

[7] Die Studien mit ihren jeweils drei Ausprägungen erinnern an die Unterscheidung, die Bernd Schönemann dargelegt hat: "Geschichte als Nutzen", "Geschichte als Bildung" und "Geschichte als Erlebnis". Vgl. dazu Bernd Schönemann, 
"Geschichtsdidaktik und Geschichtskultur," in Geschichtskultur: Theorie - Empirie - Pragmatik, eds. Bernd Mütter, Bernd Schönemann and Uwe Uffelmann (Weinheim: Beltz, 2000), 26-58, hier 47-55.

[8] Martin Nitsche, Beliefs von Geschichtslehrpersonen: Eine Triangulationsstudie (Bern: Hep Verlag, erscheint 2019). Nitsche bezeichnet Positivismus, Skeptizismus und Narrativen Konstruktivismus als "Geschichtstheoretische Überzeugungen”. Davon unterscheidet er drei "Geschichtsdidaktische Überzeugungen”: Transmission, Individueller Konstruktivismus, Sozialer Konstruktivismus. Besonders interessant in Nitsches Studie scheint mir auch, dass keine systematischen, konstruktübergreifenden Zusammenhänge zwischen den geschichtstheoretischen und -didaktischen Überzeugungen nachgewiesen werden konnten.

[9] Nicole Riedweg, Peter Gautschi and Loredana Torchetti, "Fachspezifische Überzeugungen von Geschichtslehrpersonen," in The Teaching of the History of One's Own Country, eds. Nadine Fink, Markus Furrer and Peter Gautschi (Frankfurt: Wochenschau Verlag, erscheint 2020). Mit den in dieser Studie erhobenen FragebogenDaten ließe sich auch ein Modell mit zwei Ausprägungen modellieren: Geschichte als Orientierung für Gegenwart und Zukunft / Geschichte als Konstrukt über die Vergangenheit.

[10] Identitätsstiftung gibt es auch in einem weniger ausgeprägten Maß, im Sinne eher von Traditionsbildung. Vgl. dazu Astrid Schwabe and Martin Fröhlich, "Erinnerungen an 1918 in Kiel: Schlaglichter auf die regionale Geschichtskultur im Gedenkjahr 2018," Demokratische Geschichte: Jahrbuch für Schleswig-Holstein 29 (2018): 171-197.

[11] Im Unterschied zur schulischen Geschichtsvermittlung, wo in den letzten Jahren das Konzept "Kompetenzen" dasjenige zur "Bildung" abgelöst hat, blieb in der deutschsprachigen Public History "Historische Bildung" die bestimmende Orientierung. Historisch gebildet sind - meiner Meinung nach - Menschen mit ausdifferenzierten personalen und sozialen Identitäten, die offen und neugierig dem Universum des Historischen begegnen, die über gut entwickelten Kompetenzen im Umgang mit Vergangenheit, Geschichte und Erinnerung verfügen und die darauf aufbauend die eigenen Handlungsspielräume in Gegenwart und Zukunft sehen und nutzen sowie die Chancen historischer Bildung erkennen und sich weiterhin bilden wollen.

\section{Abbildungsnachweis}

Louis Victor von Deschwanden: Kampf am Allweg (vermutlich zwischen 1800 und 1820). Mit freundlicher Genehmigung des Nidwaldner Museums (NM 514).

\section{Empfohlene Zitierweise}

Gautschi, Peter: Was Public History am meisten beeinflusst. In: Public History Weekly 7 (2019) 19, DOI: dx.doi.org/10.1515/phw-2019-13911(?).

\section{Redaktionelle Verantwortung}

Copyright (c) 2019 by De Gruyter Oldenbourg and the author, all rights reserved. This work may be copied and redistributed for non-commercial, educational purposes, if permission is granted by the author and usage right holders. For permission please contact the editor-in-chief ( $\underline{\text { see here) }}$. All articles are reliably referenced via a DOI, which includes all comments that are considered an integral part of the publication.

The assessments in this article reflect only the perspective of the author. PHW considers itself as a pluralistic debate journal, contributions to discussions are very welcome. Please note our commentary guidelines (https://public-history= weekly.degruyter.com/contribute/). 
Categories: $\underline{7}(\underline{2019}) 19$

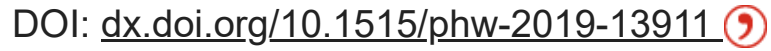

Tags: Beliefs (Grundüberzeugungen), History Teaching_(Geschichtsunterricht), Switzerland (Schweiz)

(C) Museum of Nidwalden (NM 514) 